\title{
LAS DIVERGENCIAS SOCIOECONÓMICAS TERRITORIALES COMO ALTERNATIVA METODOLÓGICA DEL ORDENAMIENTO TERRITORIAL EN MÉXICO
}

\author{
E. Propin Frejomil, A. Sánchez-Crispín y J. M. Casado Izquierdo \\ Instituto de Geografía, UNAM. Circuito Exterior \\ Ciudad Universitaria s/n. Coyoacán. México, DF \\ propinfrejomil@yahoo.com
}

\begin{abstract}
Resumen: El presente trabajo tiene como objetivo revelar las divergencias socioeconómicas de las entidades federativas de México. En una primera parte, se abordan, en forma breve, posiciones teóricas adoptadas en los trabajos de ordenamiento territorial en México. A continuación, se describen las características de los indicadores seleccionados que configuran los niveles de desarrollo y potencial socioeconómicos de las entidades federativas. Se concluye con la descripción de las divergencias territoriales, como noción cognoscitiva que posibilitó proponer criterios generales para orientar el ordenamiento territorial del país
\end{abstract}

Palabras clave: Ordenamiento territorial, México, diagnóstico socioeconómico integrado

\begin{abstract}
This paper examines the territorial socio-economic differences arising from the inconsistency between development level and socio-economic potential in each of the Mexican states. To begin with we present the theoretical positions which explain the contribution of Geography with regard to spatial management studies. Then we proceed to look at the basic features of development levels and socio-economic potential of the Mexican states; this is followed by the analysis of their territorial differences. We hope our research findings can contribute to setting up national criteria necessary to support spatial management studies in Mexico.
\end{abstract}

Key words: Spatial management, Mexico, integrated socio-economic diagnosis. 


\section{Introducción}

Los trabajos de ordenamiento territorial en México representan un movimiento institucional que ha cobrado una importancia social singular en los planos académico, docente y práctico desde 1999. La labor central desempeñada por la Secretaría de Desarrollo Social (SEDESOL) ha fusionado intereses a nivel nacional entre instancias gubernamentales y académicas como el Instituto Nacional de Estadística, Geografía e Informática (INEGI), la Secretaría de Medio Ambiente y Recursos Naturales (SEMARNAT), el Consejo Nacional de la Población (CONAPO), la Secretaría de la Presidencia y el Instituto de Geografía de la Universidad Nacional Autónoma de México (UNAM). Por su parte, las delegaciones de la SEDESOL en cada estado coordinan la elaboración de los "Programas Estatales de Ordenamiento Territorial" cuyo desarrollo es asignado a universidades o consultorías privadas ${ }^{1}$.

\section{Posiciones teóricas}

La experiencia acumulada por el Instituto de Geografía de la UNAM, como principal institución académica en el conjunto de organismos que desarrolla los trabajos de Ordenamiento Territorial (OT) en México, ha sido la base para singularizar las reflexiones y posturas teóricas siguientes:

\section{La competencia social del Ordenamiento Territorial}

Los trabajos de OT son entendidos, en lo común, como una perspectiva laboral que aglutina, o potencialmente puede hacerlo, especialistas de diversas disciplinas entre las que aparece, esencialmente, la Geografía. Esta afirmación, en lo concer-

\footnotetext{
1 La presente investigación deriva de los esfuerzos hechos durante la fase III o de "diagnóstico integrado" perteneciente a la preparación de la Guía Metodológica para los Programas Estatales de Ordenamiento Territorial, por parte del Instituto de Geografía de la Universidad Nacional Autónoma de México (UNAM) y la Secretaría de Desarrollo Social (SEDESOL) del gobierno de México. Ambas instituciones firmaron un convenio de trabajo, en 2000, con el fin de elaborar las fases III y IV de dicha guía como seguimiento de las primeras dos concebidas por el Grupo Interinstitucional (SEDESOL, el Instituto de Geografía, Estadística e Informática, la Secretaría de Medio Ambiente y Recursos Naturales, y el Consejo Nacional de Población) organizado en 1999. Este trabajo centra su atención en la revelación de las divergencias socio-económicas que distinguen a las entidades federativas de México. El contenido metodológico, incluido en la guía ya indicada, fue desarrollado en 2002 y 2003 , por organismos públicos y privados a nivel de los distintos municipios que constituyen el país.
} 
niente al desempeño geográfico, puede ser más o menos real en función de los diferentes contextos nacionales debido a la rápida expansión de los sistemas de Información Geográfica que han venido cohesionando la labor de técnicos o personas sin formación geográfica. En este orden de ideas, habría que escindir dos formas de aprehensión del mismo fenómeno que, convencionalmente, se identifican aquí en dos vertientes cognoscitivas: "el OT como coyuntura social" y "el OT como necesidad humana".

El primero es el que puede resultar novedoso porque se relaciona con las coyunturas políticas expresadas por algún organismo gubernamental. El carácter relativo de la novedad se impone como reflexión necesaria, en la medida que difiere en tiempo y espacio; México reconoce la expresión conceptual "OT" en este nuevo siglo, mientras que en otros países, como España, Francia, Países Bajos, Cuba, Venezuela o Colombia, era ya un asunto tratado en distintos momentos de la segunda mitad del siglo XX (George, 1980; Gómez, 1994; Hildebrand, 1996; Instituto Geográfico Agustín Codazzi, 1997; Instituto de Planificación Física, 1988; Méndez, 1990).

Aunado a esto, se encuentra la relatividad temporal y espacial de lo que se identifica como "problema o situación indeseada" que representa el patrón gestor del OT. Mientras que el esquema de desarrollo perspectivo de Francia centraba la atención en la descentralización de París, la prioridad se dirigía en otros contextos, como Países Bajos y Cuba (desde posiciones político-económicas diferentes) hacia el desarrollo armónico y proporcional entre sus regiones. Por su parte, países africanos como Mauritania, lo concebían como un medio para consolidar su soberanía nacional.

La segunda expresión conceptual, el OT como necesidad bumana, permite evidenciar el carácter ancestral de las acciones individuales o colectivas de organización del territorio, hayan sido o no registradas como trabajos escritos. Abordado de esta manera, el OT se entremezcla con la propia génesis del saber geográfico.

El eje temporal pasado-presente-futuro, conductor en este tipo de labores, es parte consustancial de ambas interpretaciones, tanto en su dimensión consciente y política, como en la inconsciente y humana, en la medida de que se busca eliminar la incertidumbre del futuro mediante la concepción de uno deseado y la propuesta de acciones necesarias para alcanzarlo (Gabiña, 1998).

\section{La vulnerabilidad geográfica del ordenamiento del "todo"}

El "todo" es inaprehensible por la mente humana como "síntesis suprema", de ahí que se requiera precisar el problema concreto que marcará las pautas del OT. En tal sentido, el proceso cognoscitivo entre el análisis y la síntesis desempeña la vía metodológica esencial en estos trabajos. 
Es conveniente reconocer que este proceso no es privativo del pensamiento geográfico, mucho menos del OT, sino parte sustancial de la Teoría del Conocimiento como uno de sus pares de categorías; se desmiembra la realidad para conocerla (análisis) y se integra como modelación que permite su aprehensión (síntesis). En este sentido, ambas expresiones se condicionan: la síntesis sin análisis es vacía, infundada, en la medida que el análisis desprovisto de síntesis, es conocimiento inacabado (George, op. cit.).

Lo referido, aunque entendible en su abstracción, representa una de las trampas metodológicas más insalvables en los trabajos de OT, cuando el análisis de convierte en una "enciclopédica" y costosa etapa concebida mecánicamente por la disponibilidad de bases cuantiosas de datos censales. En correspondencia, se imposibilita arribar a una imagen sintética coherente, en tanto se analizó de manera formal sin reconocer, de antemano, el contenido que debía reflejar la síntesis demandada por los propósitos iniciales del trabajo

\section{Las contradicciones entre el conocimiento geográfico y el discurso comunicativo, político e ideológico del OT}

Estas contradicciones se pueden manifestar, durante el ejercicio práctico, de las dos formas siguientes:

\section{a. Contradicciones antagónicas}

Esta distinción debe ser comprendida como las diferencias insalvables que acontecen en la precisión de lo que debe asumirse como asunto prioritario del OT. Este tipo de contradicciones puede llegar a manifestarse entre, por una parte, lo que revela el conocimiento geográfico proveniente de instituciones académicas y, por otra, lo que emana de aquellas que administran, dirigen o tienen la responsabilidad y los recursos financieros para el desarrollo socioeconómico del territorio. El problema deviene en antagónico cuando no hay acuerdos iniciales en relación a los términos, acotaciones y alcances temáticos del trabajo. Puede llegar a ocurrir que asuntos de primera relevancia para los territorios, según la parte académica, se omitan por cuestiones de inconveniencias políticas.

\section{b. Contradicciones fundamentales}

Éstas se presentan por las diferencias entre instituciones académicas y, principalmente, gubernamentales que pueden ser resueltas de maneras distintas. Con ello se relacionan, el rigor y tipo de lenguaje que ambos actores sociales utilizan en su inter- 
locución. En general, estos problemas comienzan desde la propia definición de OT donde aparecen contenidos que, en muchos casos, pueden ser calificados como "clarificadores y precisos" por el plano práctico y "confusos o imprecisos" por el académico.

En este orden de ideas, los actores investigativos deben valorar no sólo lo que aparece publicado acerca de las definiciones, alcances y contenidos del OT (Conferencia de Ministros responsables de la Ordenación del Territorio, 1983; Pujadas y Font, 1998; Hildebrand, op. cit.), sino lo que, a discreción, no se dice, aunque se caiga en el plano de apreciaciones netamente subjetivas con el propósito de entender, sobre todo, el papel de la parte académica en la relación interinstitucional que se establece. Con evidencia, la función política es la que rige la acción de intereses sociales y económicos (Conferencia de Ministros responsables de la Ordenación del Territorio, op. cit.).

Sin embargo, es conveniente reconocer que los trabajos de OT no siempre tienen un patrón idéntico en su concepción institucional. Se puede entender como práctica sociopolítica dirigida y financiada por instancias gubernamentales y como ejercicio académico vinculado con investigaciones individuales o de tesis de grado científico, que pueden llegar a ser de interés cognoscitivo para las primeras.

\section{Las soluciones conceptuales y metodológicas acordadas entre las instituciones mexicanas}

El tema de las divergencias socio-económicas territoriales se identificó como una de las partes que componen la fase de diagnóstico integrado en la guía metodológica hecha por el Instituto de Geografía de la UNAM para las entidades federativas de México (Cuadro 1). Su tratamiento se ubica, en el sentido temporal, en un lugar intermedio, en la medida que se apoya en indicadores socio-económicos utilizados en fases iniciales y orienta la labor técnica de los pasos que siguen ${ }^{2}$.

2 Esta información, en la generalidad de la experiencia recogida de las entidades federativas del país, se retoma, en algunos casos, como una referencia en los ejercicios grupales con los actores sociales de los territorios en el diseño de los escenarios futuros y, en otros, como criterio en la formulación de los objetivos y estrategias de la fase IV (Cuadro 1). En ningún caso se incorpora como contenido del Modelo de Ocupación del Territorio debido a la contradicción espacial que se establece entre las diferentes referencias territoriales del OT en México. Se decidió que el modelo aludido centrara la atención sobre las unidades de paisaje y no en la generalidad estadística de los municipios. En tal circunstancia, los resultados de las divergencias socioeconómicas territoriales se reducen a la revelación de criterios generales para acciones de corte municipal. 
Cuadro 1. Diagrama metodológico para los Programas Estatales de Ordenamiento Territorial en México.

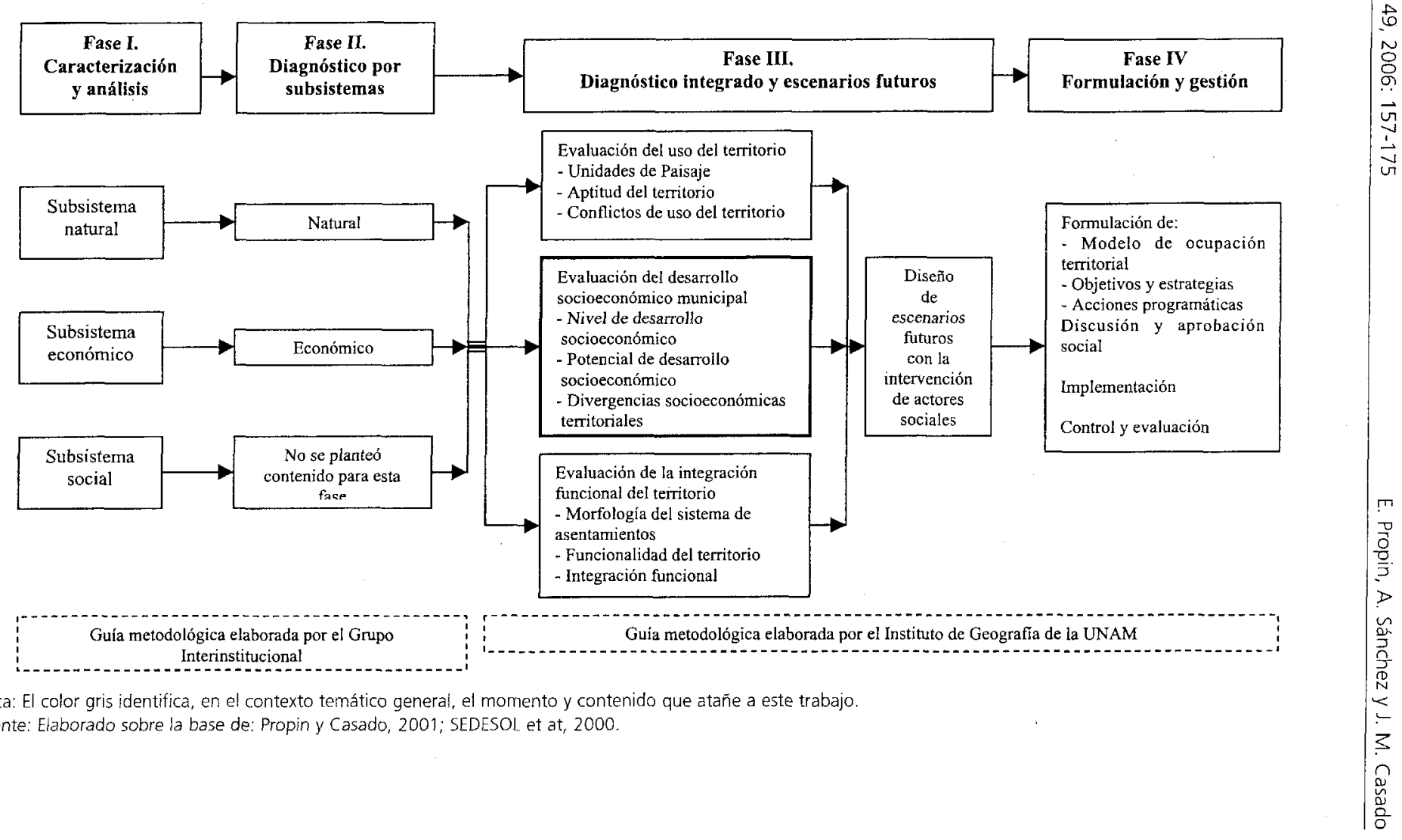


En cuanto a la comunicación conceptual entre el Instituto de Geografía de la UNAM y las otras instituciones nacionales y estatales involucradas en el proyecto, se designaron tres expresiones centrales con el fin de facilitar la interlocución.

El nivel de desarrollo socioeconómico fue definido como la desproporción relativa que yace entre los territorios estimada a partir de indicadores seleccionados. Por su parte, el potencial socioeconómico fue aprehendido como expresión medible de los factores o ventajas comparativas de un territorio. Finalmente, el código de divergencias socioeconómicas territoriales se asumió como la diferencia entre el desarrollo relativo alcanzado y las cualidades disponibles en un territorio (Propin y Casado, 2001:73, 79, 83). Este contenido representa una perspectiva metodológica distinta a las planteadas por otros estudios realizados en México acerca de las desigualdades inter-territoriales (Bejar, 1993; Bolvinik, et al, 1984; Cortes, 2000; Lustig, 1997; Salama, 1999; Székely, 1999).

Esta postura conceptual posibilitó revelar las relaciones entre la situación de desproporcionalidad relativa regional (nivel) y otra (potencial) que se asume como auxilio interpretativo para la concreción de acciones relacionadas con el aprovechamiento de las condiciones socioeconómicas de los territorios.

La disponibilidad estadística nacional, en un sentido, y la actualidad informativa que sugería el XII Censo de Población y Vivienda, levantado por INEGI en 2000 (como exigencia de las instituciones gubernamentales), en otro, fueron criterios importantes que decidieron la selección y conformación de los indicadores (Cuadro 2). En particular, se comenta, por sus relaciones temáticas complementarias, la elección y manejo de los cuatro indicadores siguientes:

-El grado de urbanización y la densidad de carreteras pavimentadas (Cuadro 2) se asumieron como mediciones del nivel de desarrollo socioeconómico debido a que el primero se correlaciona, en forma significativa, con los territorios donde se han concentrado las mayores inversiones socioeconómicas, en tanto el segundo, contempla, en forma estricta, sólo la red de ejes carreteros principales que enlaza el sistema de asentamientos urbanos principales.

-Por otra parte, la densidad de población y el coeficiente de Engel se trabajaron como parte de la medición del potencial socioeconómico de desarrollo porque el primero, en su generalidad informativa, es indicativo del total de recursos demográficos, mientras que el segundo incorpora en su formulación la red carretera y ferroviaria completa, sin discernir acerca de su categoría vial e incidencia territorial.

Las soluciones metodológicas adoptadas se dividieron en tres partes: la primera tipifica los niveles de desarrollo socioeconómico sobre la base de los cinco indicadores seleccionados y del método Z-Score; la segunda, con igual procedimiento técnico 
y número de mediciones, distingue el potencial socioeconómico de los territorios ${ }^{3}$; la tercera procede a correlacionar las dos tipologías anteriores y sintetiza las divergencias territoriales entre los contenidos referidos con anterioridad.

\section{Los niveles de desarrollo socioeconómico de las entidades federativas}

La clasificación de las entidades federativas en cinco niveles de desarrollo socioeconómico reveló los patrones regionales que se describen a continuación:

A. Nivel muy bajo. La "gran franja costera" del Pacífico entre Michoacán y Chiapas presenta los niveles más bajos, atributo que comparte con la región intermedia entre la frontera y el centro del país: Durango, Zacatecas y San Luis Potosí. Estas entidades se caracterizan por presentar porcentajes mínimos $(<51 \%)$ en el grado de urbanización ${ }^{4}$. Esta proporción predominante de personas que viven en asentamientos rurales se asocia con muy altos índices de marginación (de 0,00 a 2,36), tasas brutas reducidas de actividad económica ( $<32 \%$ ), indicativas de una muy baja participación de la población económicamente activa ocupada en relación con la total estatal, y los valores nacionales más altos (> 220\%) del coeficiente de dependencia económica que señala la mayor proporción de personas que se encuentran inactivas o desocupadas (Figura 1) y cuadro 3.

Es de interés señalar que el indicador de densidad de carreteras pavimentadas es particularmente irrelevante en México para explicar diferencias socioeconómicas regionales y fue, en correspondencia, poco significativo en la clasificación elaborada. Las condiciones naturales y la construcción de nuevas autopistas, que enlazan territorios de alto nivel a través del tránsito por otros de menor desarrollo, representan circunstancias causales que explican la aparición de altas densidades viales en estados de menor nivel como Hidalgo y la situación contraria como acontece en Chihuahua y Baja California Sur.

B. Nivel bajo. Estos estados se agrupan, mayoritariamente, hacia el Golfo de México y se extienden hacia el centro y vertiente del Pacífico a través de Guanajuato y Nayarit. Aquí se distinguen grados de urbanización bajos que oscilan entre 34\% y $59 \%$, indicativos de una notable presencia de la población residente en asentamientos rurales. Los índices altos de marginación (de 0,05 a 1,13) se relacionan con tasas de actividad económica medianamente significativas (de $31 \%$ a $36 \%$ ) y con valores muy altos del coeficiente de dependencia económica (>200\%).

\footnotetext{
3 Los valores estandarizados de tres indicadores (indice de marginación, coeficiente de dependencia económica y situación geográfica de los municipios) fueron multiplicados por (-1) por presentar una correlación negativa con el fenómeno que se examina.

4 Este trabajo asume el criterio de 15.000 y más habitantes para considerar a un asentamiento humano como urbano en el contexto de México (Unikel, 1976).
} 
Cuadro 2. Indicadores seleccionados para el cálculo de los niveles de desarrollo y potencial socioeconómicos.

\section{NIVELES DE DESARROLLO SOCIOECONÓMICO}

\begin{tabular}{|c|c|c|}
\hline a. Grado de urbanización & $\mathrm{GU}=\frac{\mathrm{Pu}}{\mathrm{Pt}} \times 100$ & $\begin{array}{l}\text { GU: grado de urbanización (\%) } \\
\text { Pu: población urbana estatal; se entiende como el total de población que reside en asentamientos de } 15000 \text { y más } \\
\text { habitantes. } \\
\text { Pt: población total estatal }\end{array}$ \\
\hline b. Índice de marginación & & $\begin{array}{l}\text { Se adoptará la información elaborada por CoNAPO como síntesis de atributos significativos concernientes a la condiciones } \\
\text { de vida de la población, relacionada con la política demográfico-territorial del pais; se adopta el indice de marginación - IM } \\
\text { (expresión cuantitativa) en lugar del grado (expresión cualitativa) con fines de desarrollar el procesamiento metodológico } \\
\text { cuantitativo posterior }\end{array}$ \\
\hline $\begin{array}{l}\text { c. Tasa bruta de actividad } \\
\text { económica }\end{array}$ & TBAE $=\frac{\text { PEAO }}{\mathrm{Pt}} \times 100$ & $\begin{array}{l}\text { TBAE: tasa bruta de actividad económica (\%) } \\
\text { PEAo: población económicamente activa ocupada estatal } \\
\text { Pt: población total estatal }\end{array}$ \\
\hline \multirow[t]{2}{*}{$\begin{array}{l}\text { d. Coeficiente de } \\
\text { dependencia económica }\end{array}$} & $\mathrm{CDE}=\frac{\mathrm{P} \times 12+\mathrm{PEAd}+\mathrm{PEI}}{\mathrm{PEA0}} \times 100$ & $\begin{array}{l}\text { CDE: coeficiente de dependencia económica }(\%) \\
\text { Psti: población estatal con menos de } 12 \text { años* } \\
\text { PEAd: población estatal económicamente activa desocupada } \\
\text { PEl: población estatal económicamente inactiva** } \\
\text { PEAO: pobobación estatal económicamente ocupada }\end{array}$ \\
\hline & \multicolumn{2}{|c|}{$\begin{array}{l}\text { Aclaraciones estadisticas: } \\
12 \text { años. } \\
\text { "* Las redución con menos de } 12 \text { años, se calcula a partir de la diferencia con la población total; se considera que la población con nivel de actividad (activa o inactiva) no indicada quedan, inevitablemente, fuera del cálculo. }\end{array}$} \\
\hline $\begin{array}{l}\text { e. Densidad de carreteras } \\
\text { pavimentadas }\end{array}$ & DCP $=\frac{\operatorname{Lcp}}{\mathrm{S}}$ & $\begin{array}{l}\left.\text { DCP: densidad de carreteras pavimentadas ( } \mathrm{km} / 100 \mathrm{~km}^{2}\right) \\
\text { LCp: Iongitud de carreteras pavimentadas en el estado } \\
\text { S: superficie estatal }\end{array}$ \\
\hline \multicolumn{3}{|c|}{ J1. POTENCIAL SOCIOECONOMICO DE DESARROLLOO } \\
\hline $\begin{array}{l}\text { a. Situación geográfica de } \\
\text { los estados }\end{array}$ & $S G M=L C_{(m-c)}$ & $\begin{array}{l}\text { SGM: situación geográficá de los estados } \\
\text { LC } \\
\text { (m-t: menor distancia aérea (segmento rectilineo) entre la capital estatal y Cd. de México }(\mathrm{km})\end{array}$ \\
\hline b. Densidad de población & $\mathrm{DP}=\frac{\mathrm{Pt}}{\mathrm{S}}$ & $\begin{array}{l}\left.\text { OP: densidad do población (hab. } / \mathrm{km}^{2}\right) \\
\text { Pt: población total estatal } \\
\text { S: superficie estatal }\left(\mathrm{km}^{2}\right)\end{array}$ \\
\hline $\begin{array}{l}\text { c. Grado de calificación de } \\
\text { la población }\end{array}$ & $G C P=\frac{P_{12}>31 ; p+P_{15}>3 \text { 3e: }+P_{12>31}}{P_{12}} \times 100$ & 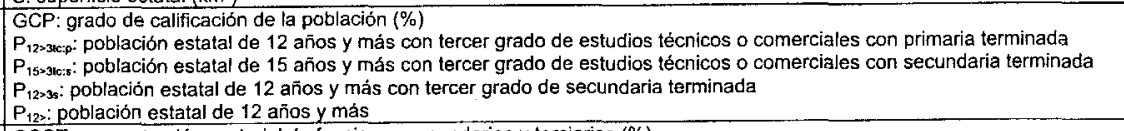 \\
\hline $\begin{array}{l}\text { d. Concentración sectorial } \\
\text { de las funciones } \\
\text { secundarias y terciarias }\end{array}$ & $\operatorname{COST}=\frac{\text { PEAOS }+ \text { PEAot }}{\text { PEAO }} \times 100$ & $\begin{array}{l}\text { COST: concentración sectorial de funciones secundarias y terciarias (\%) } \\
\text { PEAos: población económicamente activa ocupada en el sector secundario } \\
\text { PEAot: población económicamente activa ocupada en el sector terciario } \\
\text { PEAo: población económicamente activa ocupada }\end{array}$ \\
\hline $\begin{array}{l}\text { e. Coeficiente de } \\
\text { suficiencia de la red vial }\end{array}$ & $K=\frac{L c+L f}{\sqrt{S \times P t}} \times 100$ & $\begin{array}{l}\text { K: coeficiente de suficiencia de la red vial (se reconoce también como coeficiente de Engel) } \\
\text { Lc: longitud de la red vial carretera en el estado (pavimentada, terraceria y brecha) }(\mathrm{km}) \\
\text { Li: longitud de vias ferreas en el estado }(\mathrm{km}) \\
\text { S: superficie estatal }\left(\mathrm{km}^{2}\right) \\
\text { Pt: población total estatal }\end{array}$ \\
\hline
\end{tabular}


C. Nivel medio. Caracteriza a los estados fronterizos del centro-norte, incluso Sinaloa, aunque se presenta en casos aislados como Yucatán y Jalisco o en forma asociada como Querétaro y Tlaxcala, en vecindad con el Distrito Federal. El predominio de la población urbana, con valores medios y altos, diferencia a este grupo (50\% $85 \%$ ), salvo el caso del estado de Tlaxcala que contradictoriamente reporta un comportamiento más favorable en los restantes indicadores a pesar de reportar una población rural predominante. Este grupo de entidades se asocia con menores índices de marginación (de 0,80 a 0,85), Yucatán aparece como el más marginado, y coeficientes de dependencia económica menos significativos (de 166\% a 193\%).

D. Nivel alto. Las entidades con niveles altos se localizan en forma discontinua, salvo los casos de las delimitadas en la península de Baja California y la vecindad geográfica Nuevo León-Tamaulipas; en general se presentan en asociación espacial con estados de niveles medios como Colima y Aguascalientes, respecto a Jalisco, y Quintana Roo en relación con Yucatán. Aquí se identifican grados significativos de urbanización (de 70\% a 89\%) excepto el caso de Morelos que se comporta como Tlaxcala en el grupo anterior. Sin incluir el caso del Distrito Federal, este grupo es el de menor marginación (de $-0,55$ a $-1,50$ ), tasas brutas mayores de actividad económica (de 34\% a 39\%) y dependencia económica menor (de $150 \%$ a $193 \%$ ).

Figura 1. Nivel de desarrollo socioeconómico de las entidades federativas.

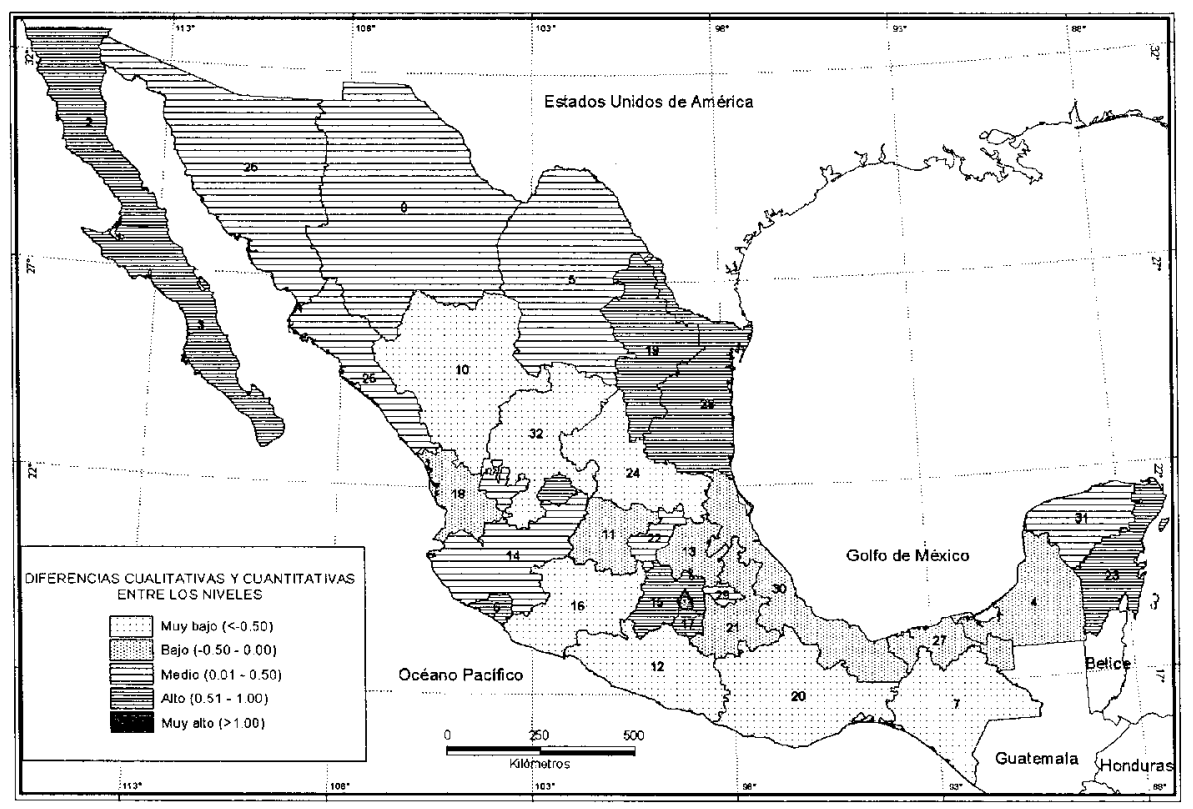


Cuadro 3. Comportamiento cuantitativo de indicadores seleccionados para medir el nivel de desarrollo socioeconómico de las entidades federativas

\begin{tabular}{|c|c|c|c|c|c|c|c|}
\hline Nivel & $\begin{array}{l}\text { Indice } \\
\text { medio }\end{array}$ & Nombre & $\begin{array}{c}\text { Grado de } \\
\text { urbanización, } \\
2000(\%)\end{array}$ & $\begin{array}{l}\text { Índice de } \\
\text { marginación, } \\
1995\end{array}$ & $\begin{array}{l}\text { Tasa bruta } \\
\text { de actividad } \\
\text { económica, } \\
2000(\%)\end{array}$ & $\begin{array}{l}\text { Coeficiente de } \\
\text { dependencia } \\
\text { económica, } \\
2000(\%)\end{array}$ & $\begin{array}{c}\text { Densidad } \\
\text { de carreteras } \\
\text { pavimentadas, } \\
1999 \\
\left(\mathrm{Km} / 100 \mathrm{Km}^{2}\right) \\
\end{array}$ \\
\hline Muy bajo & $-1,56$ & 32. Zacatecas & 33,56 & 0,60 & 26,12 & 281,73 & 13,42 \\
\hline Muy bajo & $-1,31$ & 12. Guerrero & 39,00 & 1,91 & 28,84 & 245,84 & 16,41 \\
\hline Muy bajo & $-1,22$ & 20. Oaxaca & 22,47 & 1,85 & 31,02 & 221,61 & 13,42 \\
\hline Muy bajo & $-1,10$ & 7. Chiapas & 28,61 & 2,36 & 30,77 & 224,07 & 26,14 \\
\hline Muy bajo & $-0,70$ & 16. Michoacán & 43,92 & 0,39 & 30,78 & 223,97 & 15,73 \\
\hline Muy bajo & $-0,67$ & 10. Durango & 50,94 & 0,00 & 30,62 & 225,54 & 9,01 \\
\hline Muy bajo & $-0,67$ & 24. San Luis Potosi & 47,20 & 0,76 & 31,13 & 220,49 & 17,30 \\
\hline Bajo & $-0,48$ & 27. Tabasco & 34,22 & 0,67 & 31,73 & 214,26 & 33,58 \\
\hline Bajo & $-0,43$ & 13. Hidalgo & 30,60 & 1,00 & 32,60 & 206,14 & 36,50 \\
\hline Bajo & $-0,41$ & 21. Puebla & 44,43 & 0,80 & 32,81 & 204,07 & 24,01 \\
\hline Bajo & $-0,40$ & 30. Veracruz & 41,70 & 1,13 & 34,02 & 193,35 & 21,30 \\
\hline Bajo & $-0,29$ & 4. Campeche & 52,96 & 0,78 & 35,23 & 183,29 & 6,56 \\
\hline Bajo & $-0,28$ & 18. Nayarit & 41,82 & 0,05 & 34,65 & 187,95 & 11,51 \\
\hline Bajo- & 0,26 & 11. Guanajuato & 58,37 & 0,13 & 31,31 & 218,37 & 27,64 \\
\hline Medio & 0,01 & 25. Sinaloa & 52,65 & $-0,21$ & 34,70 & 187,36 & 17,37 \\
\hline Medio & 0,09 & 22. Querétaro & 50,97 & $-0,19$ & 34,18 & 191,62 & 28,77 \\
\hline Medio & 0,21 & 31. Yucatán & 58,85 & 0,80 & 37,30 & 167,63 & 22,34 \\
\hline Medio & 0,37 & 26. Sonora & 71,84 & $-0,85$ & 36,56 & 172,63 & 6,01 \\
\hline Medio & 0,37 & 8. Chihuahua & 75,42 & $-0,78$ & 36,61 & 172,04 & 4,74 \\
\hline Medio & 0,46 & 5. Coahuila & 84,28 & $-1,18$ & 35,80 & 178,31 & 6,39 \\
\hline Medio & 0,47 & 29. Tlaxcala & 38,59 & $-0,23$ & 34,13 & 192,19 & 63,45 \\
\hline Medio & 0,50 & 14. Jalisco & 71,36 & $-0,60$ & 37,37 & 166,95 & 13,68 \\
\hline Alto & 0,53 & 17. Morelos & 59,39 & $-0,55$ & 35,42 & 181,59 & 39,82 \\
\hline Alto & 0,56 & 28. Tamaulipas & 78,62 & $-0,58$ & 36,80 & 170,83 & 16,13 \\
\hline Alto & 0,60 & 3. Baja California Sur & 63,11 & $-0,84$ & 39,86 & 150,00 & 4,88 \\
\hline Alto & 0,61 & 2. Baja California & 83,97 & $-1,27$ & 36,44 & 172,90 & 9,58 \\
\hline Alto & 0,64 & 15. México & 72,93 & $-0,74$ & 34,07 & 192,74 & 44,19 \\
\hline Alto & 0,67 & 23. Quintana Roo & 73,17 & $-0,22$ & 39,86 & 150,32 & 11,86 \\
\hline Alto & 0,67 & 1. Aguascalientes & 72,86 & $-1,05$ & 35,06 & 184,40 & 34,81 \\
\hline Alto & 0,67 & 6. Colima & 70,16 & $-0,71$ & 36,80 & 171,01 & 28,80 \\
\hline Alto & 0,95 & 19. Nuevo León & 88,77 & $-1,50$ & 38,54 & 158,71 & 11,27 \\
\hline Muy Alto & 1,39 & 9. Distrito Federal & 98,83 & $-1,74$ & 41,63 & 139,53 & 10,09 \\
\hline
\end{tabular}

Fuente: Elaborado sobre la base de: INEGI, 2001; CONAPO-PROGRESA, 1998; SCT, 1999.

E. Nivel muy alto. Distingue sólo al Distrito Federal con los grados mayores de urbanización y tasa bruta de actividad económica del país e índice de marginación y coeficiente de dependencia económica menores.

\section{El potencial socioeconómico de las entidades federativas}

Los patrones regionales que revelan los niveles del potencial socioeconómico de las entidades federativas difieren, en forma sustancial, de la clasificación anterior, 
excepto en las situaciones extremas entre Chiapas-Oaxaca, en el inferior, y Distrito Federal-Tlaxcala-México, en los estratos superiores. El carácter relativamente homogéneo de comportamiento se evidencia entre catorce estados con bajo potencial y doce en situación intermedia. Entre los últimos se distingue el eje Querétaro-HidalgoGuanajuato-Aguascalientes-Jalisco-Colima, grupo al que se une Morelos, y la asociación espacial Coahuila-Nuevo León-Tamaulipas (Figura 2 y Cuadro 4). Los de bajo potencial se agrupan en dos patrones regionales diferenciados: el primero, mediante vecindades con los de niveles intermedios en la porción norte del país y el segundo, menos favorable, con los de muy bajo potencial en la porción sur.

El comportamiento irregular de dos indicadores merecen un comentario particular. El coeficiente de suficiencia de la red vial, también reconocido como de Engel, se mostró poco significado en la clasificación; la relación entre las longitudes viales terrestres y las dimensiones territoriales y poblacionales informaron de suficiencias viales en territorios de muy bajo potencial socioeconómico como en Chiapas y Tabasco y de insuficiencias en otros de mayor potencial como Nuevo León y el Distrito Federal.

Por su parte, el indicador de distancia a la Ciudad de México, seleccionado bajo la hipótesis de que a mayor cercanía y vecindad geográfica con la capital, los estados

Figura 2. Potencial socioeconómico de las entidades federativas.

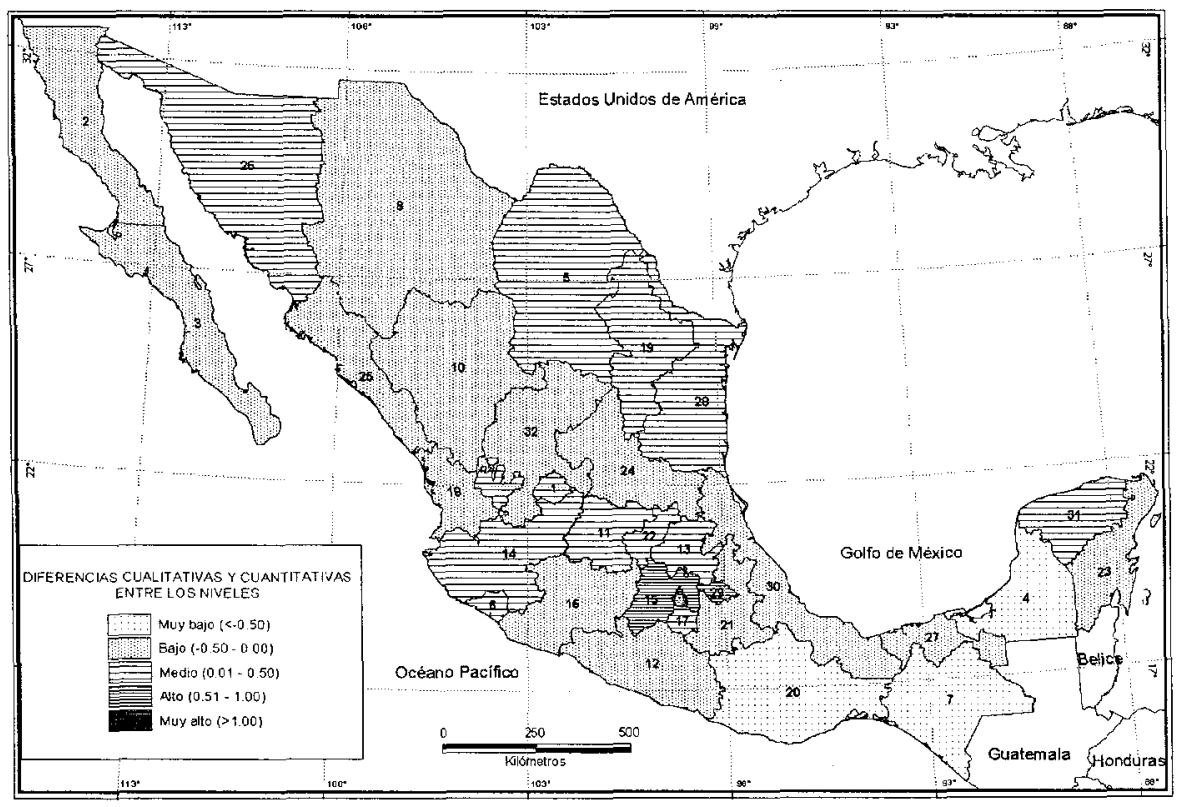


Cuadro 4. Comportamiento cuantitativo de los indicadores seleccionados para medir el potencial socioeconómico de las entidades federativas

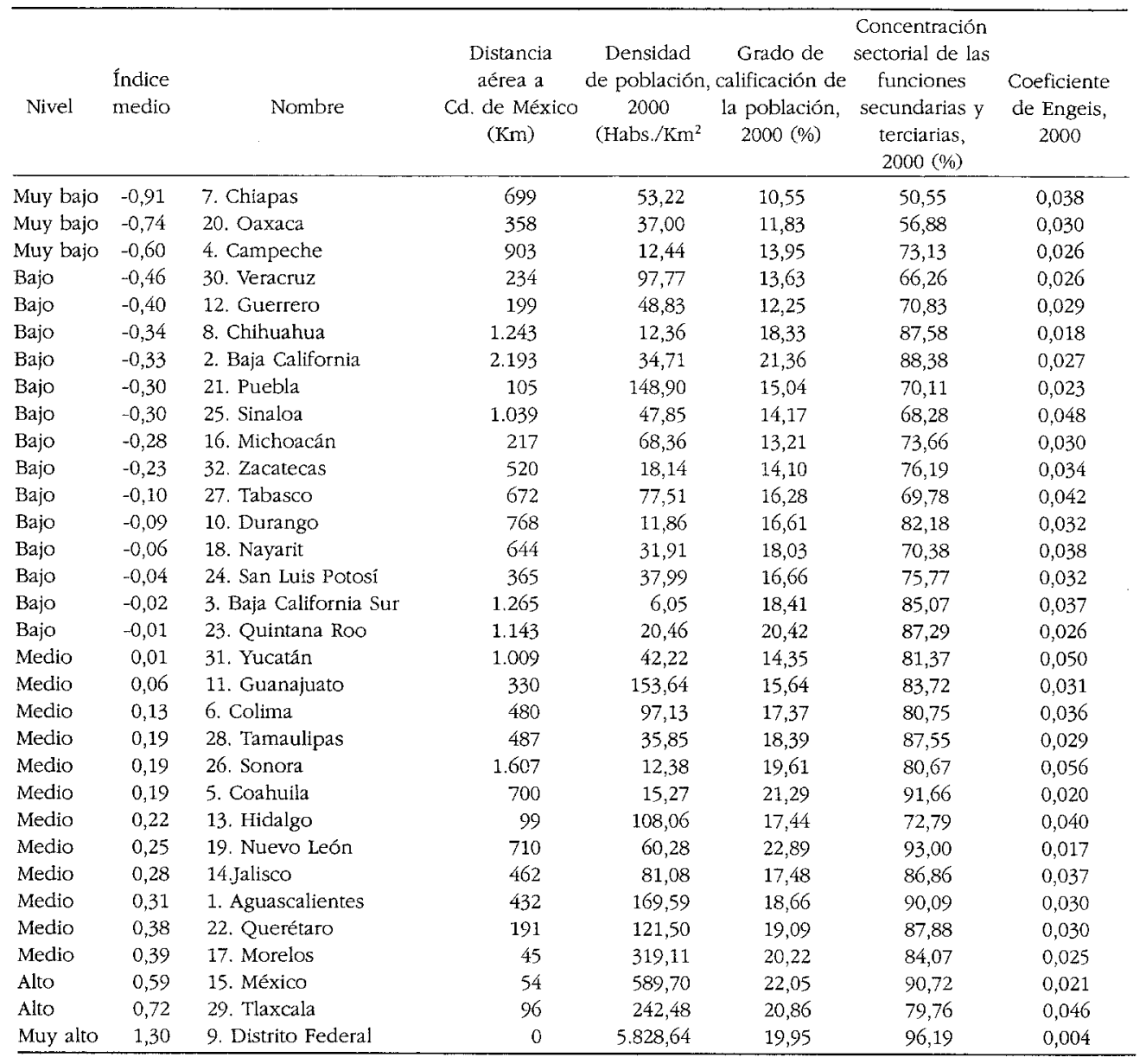

Fuente: Elaborado sobre la base de: INEGI, 2001; SCT, 1999; SCT 2000.

poseen mayores ventajas socioeconómicas, sobre todo considerando la macrocefalia demográfica y económica del país, se presentó en forma parcialmente irregular. La hipótesis es válida, salvo en el caso de Puebla que se ubicó entre los estados de bajo potencial socioeconómico debido al comportamiento de los restantes indicadores y en los estados de la frontera norte, vecinos de Nuevo León, y en Sonora y Yucatán donde los indicadores concernientes al grado alto de calificación de la población y la acentuada concentración de funciones secundarias y terciarias marcan el rango del potencial por encima de las densidades bajas de población y la lejanía respecto a la Ciudad de México. 
Las entidades federativas quedaron clasificadas en los niveles que se describen a continuación:

A. Muy bajo. Se integra por tres estados: Chiapas, Oaxaca y Campeche que se asemejan por sus índices de potencial socioeconómico, inferior a - 0,50. La distancia con respecto a la Ciudad de México y las densidades de población que reportan tienen valores desiguales entre sí. En contraste, los indicadores referidos a población de doce años y más con nivel de educación secundaria o equivalente, la PEA no agrícola y el coeficiente de Engel son parecidos, con valores que se encuentran entre los más bajos del país.

B. Bajo. Es el grupo de estados más numeroso, catorce en total, con una distribución geográfica irregular que incluye desde la península de Baja California (ambas entidades) hasta Quintana Roo. En estas circunstancias, el valor que indica la distancia con respecto a la Ciudad de México varía significativamente desde mas de 2.000 kilómetros para Baja California hasta menos de 200 kilómetros en los casos de Puebla y Guerrero. La densidad de población también tiene cifras que fluctúan: $6 \mathrm{hab} / \mathrm{km}^{2}$ en Baja California Sur y casi 150 en Puebla. Los valores que cohesionan la presencia de determinados estados en este nivel corresponden con la población de doce años y más con nivel de secundaria o equivalente y la PEA secundaria y terciaria; en todos los casos existe un predominio de la PEA ocupada en actividades secundaria y terciaria en relación con la total de cada estado.

C. Medio. Este nivel caracteriza a doce de las entidades del país. La distribución territorial de éstas también es irregular: cuatro estados en el norte (Sonora, aislado respecto a los otros tres y la terna Coahuila- Nuevo León-Tamaulipas, ya referida en el caso del nivel de desarrollo socioeconómico); siete estados en la porción central del país (Hidalgo, Querétaro, Guanajuato, Jalisco, Aguascalientes y Colima, en contigüidad territorial, y Morelos, separado físicamente del resto del conjunto). Finalmente, se incluye el caso de Yucatán localizado en el extremo este de México. Los indicadores que consolidan la presencia de estas entidades en este nivel se refieren a la PEA no agrícola que supera, en general, el $80 \%$ del total y el grado de calificación de la población, con valores por encima de $14 \%$.

D. Alto. Sólo dos entidades del centro del país quedan en este nivel: México y Tlaxcala que se caracterizan por la cercanía con respecto a la Ciudad de México, los altos valores de la densidad de población (mayores que $242 \mathrm{hab} / \mathrm{km}^{2}$ ), el alto grado de calificación de la población y la acentuada concentración de actividades secundarias y terciarias.

E. Muy alto. En esta circunstancia, aparece el Distrito Federal. El potencial socioeconómico de este espacio, donde se asienta la capital del país, es el mayor de México con una densidad de población muy alta (por encima de los 5.000 habs $/ \mathrm{Km}^{2}$ ) y valo- 
res elevados en el grado de calificación de la población y la concentración de funciones secundarias y terciarias.

\section{La dimensión nacional de las divergencias socioeconómicas territoriales}

La tipología final trabajada como síntesis de los niveles de desarrollo y el potencial socioeconómico de las entidades federativas revela tres tipos de correlaciones territoriales (Figuras 3 y 4 ):

\section{Divergencia territorial positiva}

El nivel de desarrollo socioeconómico alcanzado se muestra por encima o a la par de los factores potenciales de los territorios. Esta situación es la que distingue a diecisiete de las entidades federativas que se distribuyen mediante patrones regionales regulares: la frontera norte, los estados vecinos del Distrito Federal y Jalisco y el binomio Yucatán-Quintana Roo. Aquí no aparece el potencial socioeconómico como favorecedor del nivel de desarrollo económico en tanto reúne a las entidades que han recibido, en distintos momentos, un fuerte impulso inversionista en la industria y el turismo.

La concentración del sector secundario maquilador en los estados de la frontera norte, los parques industriales o determinadas industrias básicas en las proximidades de Guadalajara y en las localidades de Aguascalientes y Querétaro, y la atención prioritaria en el terciario o terciario-industrial en Morelos, Yucatán y Quintana Roo se soportan más por los intereses económicos que sobre condiciones de la concentración territorial y grado de calificación de la población. Sin embargo, esta circunstancia, positiva en un sentido, posee una limitante relacionada con el potencial socioeconómico: buena parte de los empleos que genera no exigen una alta calificación de la población y son de baja remuneración.

En situación extrema se halla el Distrito Federal, vértebra fundamental de la economía nacional y punto de referencia, en sentido centrípeto o centrífugo, de los intercambios de bienes, personas y satisfactores del sector terciario con todas las regiones del país. La presencia añeja de la industria, la disponibilidad de servicios de todo tipo, la oferta de transporte y la muy intensa actividad comercial que se despliegan en el territorio del Distrito Federal son la base que genera esta divergencia territorial positiva, la mas alta de México. 
Figura 3. Divergencias territoriales entre los niveles de desarrollo y de potencial socioeconómico de las entidades federativas.

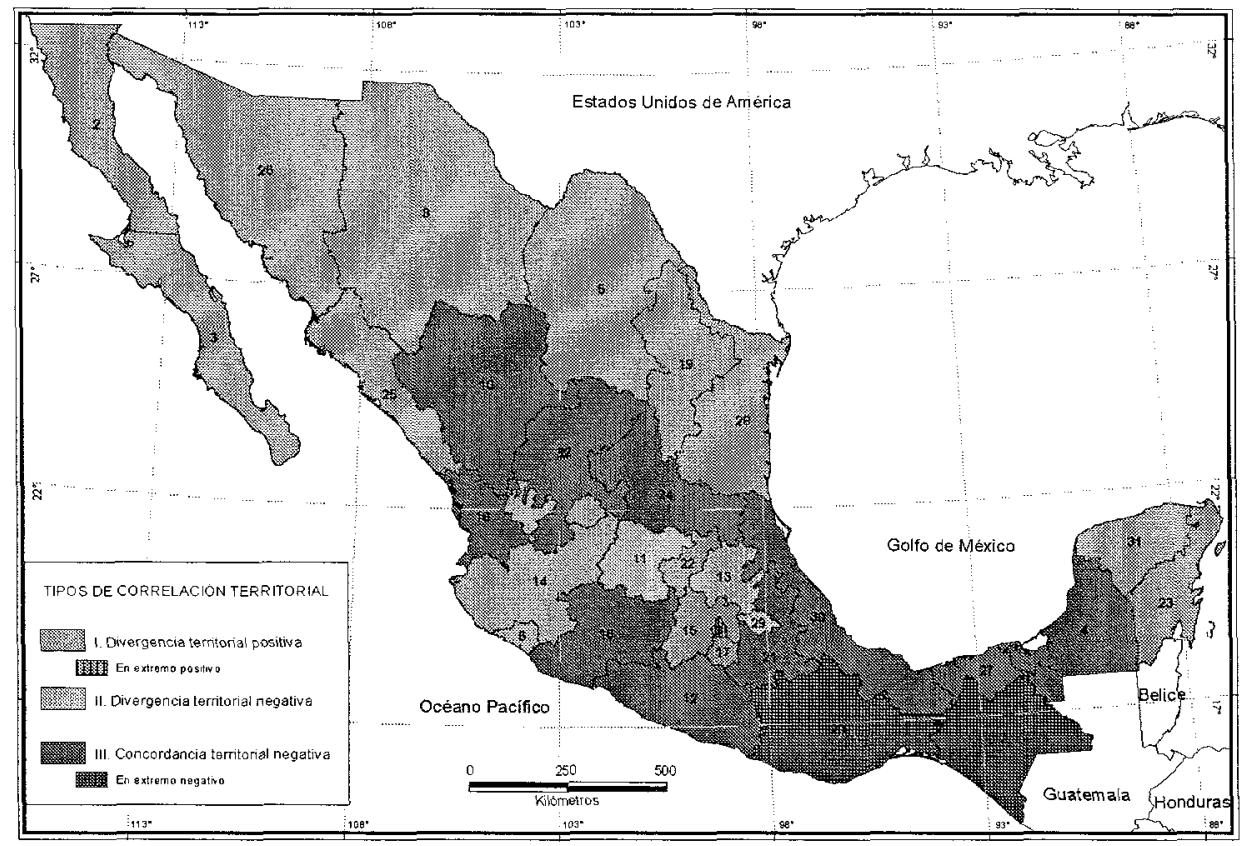

\section{Divergencia territorial negativa}

El nivel de desarrollo está por debajo del que cabría esperar, de acuerdo con el potencial socioeconómico; el atraso relativo deriva de la infrautilización de ese potencial, no de su inexistencia. Representa la situación más favorable para impulsar determinados planes de desarrollo regional donde se aproveche la cercanía a la ciudad capital del país, el grado de calificación de la población, la concentración existente de actividades secundarias y terciarias y su relativa suficiencia de la red vial; circunstancias halladas en los estados de Guanajuato, Hidalgo y Tlaxcala.

\section{Concordancia territorial negativa}

Estos territorios se caracterizan por un nivel bajo de desarrollo, unido a la presencia de un potencial socioeconómico débil; por lo que resulta más difícil diseñar estrategias de desarrollo en este tipo de estados. Tal situación es la que caracteriza doce entidades federativas. El impulso económico gestado en determinados lugares de éstas, como el caso de las economías del petróleo (Veracruz, Tabasco y 
Figura 4. Tipología para la clasificación de entidades federativas

\begin{tabular}{|c|c|c|c|c|c|c|}
\hline \multirow{7}{*}{ 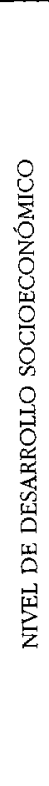 } & & \multicolumn{5}{|c|}{ NIVEL DE POTENCIAL SOCIOECONÓMICO } \\
\hline & & $\begin{array}{c}\text { Muy bajo } \\
(<-0,50)\end{array}$ & Bajo $(-0,50-0,00)$ & Medio $(0,01-0,50)$ & Alto $(0,51-1,00)$ & Muy alto $(>1,00)$ \\
\hline & Muy bajo $(<-0,50)$ & $\begin{array}{l}\text { 7. Chiapas } \\
\text { 20. Oaxaca }\end{array}$ & $\begin{array}{l}\text { 10. Durango } \\
\text { 12. Guerrero } \\
\text { 16. Michoacán } \\
\text { 24. S.L. Potosí } \\
\text { 32. Zacatecas }\end{array}$ & & & \\
\hline & Bajo $(-0,50-0,00)$ & 4. Campeche & $\begin{array}{l}\text { 18. Nayarit } \\
\text { 21. Puebla } \\
\text { 27. Tabasco } \\
\text { 30. Veracruz }\end{array}$ & $\begin{array}{l}\text { 11. Guanajuato } \\
\text { 13. Hidalgo }\end{array}$ & & \\
\hline & Medio $(0,01-0,50)$ & & $\begin{array}{l}\text { 8. Chihuahua } \\
\text { 25. Sinaloa }\end{array}$ & $\begin{array}{l}\text { 5. Coahuila } \\
\text { 14. Jalisco } \\
\text { 22. Querétaro } \\
\text { 26. Sonora } \\
\text { 31. Yucatán }\end{array}$ & 29. Tlaxcala & \\
\hline & Alto $(0,51-1,00)$ & & $\begin{array}{l}\text { 2. Baja California } \\
\text { 3. Baja California Sur } \\
\text { 23. Quintana Roo }\end{array}$ & $\begin{array}{l}\text { 1. Aguascalientes } \\
\text { 6. Colima } \\
\text { 17. Morelos } \\
\text { 19. Nuevo León } \\
\text { 28. Tamaulipas }\end{array}$ & 15. México & \\
\hline & Muy alto $(>1,00)$ & & & & & 9. Distrito Federal \\
\hline
\end{tabular}

Fuente: Elaborado sobre la base de las figuras 1 y 2.

Campeche) y del turismo (Guerrero), entre otros ejemplos, no ha significado un impacto regional significativo.

En particular, el nivel y potencial socioeconómico muy bajo de Chiapas y Oaxaca, los menos favorecidos, se relaciona con la presencia de los principales núcleos de grupos indígenas y una dispersión alta de la población rural, lo cual emerge como obstáculo para el aprovechamiento de los recursos naturales locales y, en consecuencia, para una diversificación e impulso de determinados tipos de economía.

\section{Conclusiones}

Las divergencias socioeconómicas reveladas representan una imagen que demuestra las condiciones disimiles desde las que parten los diferentes estados de México para enfocar los objetivos de sus respectivos ordenamiento territoriales. Si bien, la SEDESOL los ha tratado en forma igual en los procesos de contratación y asignación de recursos financieros, es un hecho que un conjunto de entidades se encuentran en situaciones de desventaja relativa o poseen condiciones menos propicias para impulsar este tipo de trabajo. 
Las entidades con divergencias territoriales, tanto positivas como negativas, enfrentan, en sus ordenamientos estatales, problemas diversos relacionados con la funcionalidad de su base socioeconómica. En este sentido, las dependencias externas de sus economías secundarias y terciarias, como la industria maquiladora de exportación y el turismo, o el débil aprovechamiento de su potencial humano deberían ser contenidos temáticos a abordar en sus respectivos estudios de OT.

Por otra parte, las entidades con concordancia territorial negativa parten de circunstancias contextuales opuestas, en las que la infraestructura socioeconómica carente o débil, la complejidad étnica y los conflictos sociales internos emergen como limitantes que marcan pautas importantes en sus estrategias de desarrollo futuro. Sin embargo, estos estados son los que poseen el potencial de recursos naturales más alto del país. En este sentido, deberian ocupar un lugar de atención preferencial en las gestiones de OT por parte de las instancias nacionales

\section{Bibliografía}

Bastié, J. (1988) ¿Es un mito la ordenación del territorio?. Reflexiones sobre la ordenación territorial de las grandes metrópolis. México. Instituto de Geografía de la UNAM.

Bejar, R. (1993) Población y desigualdad social en México. Cuernavaca. Centro Regional de Investigaciones Multidisciplinarias, UNAM.

Bolvinik, J., et al (1984) La desigualdad en México. México. Siglo XXI

CONAPO-PROGRESA (1998) Indice de marginación, 1995, anexo II. http://www. conapo.gob.mx/m_en_cifras/principal.ht $\mathrm{ml}$

Conferencia de Ministros responsables de la Ordenación del Territorio (1983) Carta Europea de la Ordenación del Territorio. Bruselas.

Cortes, F. (2000) Procesos sociales y desigualdad económica en México. México. Siglo XXI.
Duque, R. (2000) El ordenamiento territorial ante la desigualdad regional y los riesgos naturales. México. Ingeniería Civil. pp. 24-28.

Gabiña, J. (1998) Prospectiva y ordenación del territorio: bacia un proyecto de futuro. Barcelona. Marcombo.

George, P. (1980) Geografia Activa. Barcelona.

Godet, M. (1995) De la anticipación a la acción: manual de prospectiva y estrategia. México. Alfaomega.

Gómez, D. (1994) Ordenación del territorio. una aproximación desde el medio fisico. Madrid.

Hildebrand, A. (1996) Politica de ordenación del territorio en Europa. Sevilla. Universidad de Sevilla - Consejería de Obras Públicas y transportes de la Junta de Andalucía.

INEGI (2001) Sistema Municipal de Bases de Datos (SIMBAD). XII Censo General de 
Población y Vivienda, 2000. http://www. inegi.gob.mx

Instituto de Planificación Física (1988) Evento Nacional de Planificación Regional $y$ Urbana (libro de resúmenes). La Habana. Instituto de Planificación Física de Cuba.

Instituto Geográfico Agustín Codazzi (1997) Bases conceptuales y guia metodológica para la formulación del plan de ordenamiento territorial departamental. Bogotá. Editorial Linotipia.

Lustig, N. C. (1997) México: evolución económica, pobreza y desigualdad. Washington. Banco Interamericano de Desarrollo.

Méndez, E. (1990) Gestión ambiental y ordenación del territorio. Mérida (Venezuela). Universidad de Los Andes.

Propin, E. y J. M. Casado (2001) Evaluación del desarrollo socioeconómico municipal". En VV.AA. Guia conceptual y metodológica para el diagnóstico integrado del sistema territorial. México. Instituto de Geografía, UNAM. pp. 73-85.
Pujadas, R. y J. Font (1998) Ordenación y planificación territorial. Madrid. Sintesis.

Salama, P. (1999) Globalización, desigualdades territoriales y salariales. Problemas del Desarrollo: Revista Latinoamericana de Economia. Vol. XXX, 117, pp. 117-139.

SCT (1999) Longitud y caracteristicas de la red de carreteras por entidad federativa. http://www.sct.gob.mx

SCT (2000) Vias férreas existentes por entidad federativa. http://www.sct.gob.mx

SEDESOL et al. (2000) Términos de referencias generales para la elaboración del Programa Estatal de Ordenamiento Territorial. México.

Székely, M. (1999) La desigualdad en México: una perspectiva internacional. Banco Interamericano de Desarrollo. México.

Unikel, L. (1976) El desarrollo urbano de México. Diagnóstico e implicaciones futuras. El Colegio de México. México. 